\title{
ASYMPTOTIC VALUES AND THE GROWTH OF ANALYTIC FUNCTIONS IN SPIRAL DOMAINS
}

\author{
J. E. BRENNAN AND A. L. Volberg
}

\begin{abstract}
In this note we present a simple proof of a theorem of Hornblower which characterizes those functions analytic in the open unit disk having asymptotic values at a dense set in the boundary. Our method is based on a kind of $\bar{\partial}$-mollification and may be of use in other problems as well.
\end{abstract}

\section{Introduction}

It is a well-known fact that if $f$ is analytic in the open unit disk $D$ and if $|f|$ is subject to a sufficiently strong growth restriction, then $f$ has radial limits almost everywhere on $\partial D$. In 1963, however, G. R. MacLane [14] extended that principle to a much larger class of functions, where radial limits are completely inadequate for a description of boundary behavior. A function $f$ analytic in $D$ is said to have an asymptotic value $\lambda$ at the point $\zeta_{0} \in \partial D$ if there exists an arc $\Gamma$ lying in $D$ with one endpoint at So such that

$$
f(z) \rightarrow \lambda \text { as } z \rightarrow \zeta_{0} \text { along } \Gamma .
$$

Here, $\lambda=\infty$ is allowed and it is entirely possible for a given $f$ to have more than one asymptotic value at a single point $\zeta_{0} \in \partial D$ (cf.[14], [16]). The MacLane class $\mathcal{A}$ consists of those nonconstant analytic functions having asymptotic values at a dense set in $\partial D$.

The principal goal of MacLanc's work is to obtain conditions on a. given $f$ sufficient to guarantee that $f \in \mathcal{A}$, and his results are based on the following simple fact: If $f$ is analytic in $D$ and $\gamma$ is an arc on $\partial D$ then either

(i) $f$ has asymptotic values at a dense set in $\gamma$ or;

(ii) there exists a sequence of Koebe arcs $\gamma_{n}$ tending to a nontrivial subarc $\gamma^{\prime}$ of $\gamma$ such that $|f(z)|=c>0$ on $\gamma_{n}, n=1,2, \ldots$ 
In particular, if as usual,

$$
M(r)=M(r, f)=\sup _{|z|=r}|f(z)|
$$

it can be shown (cf. [14]) that $f \in \mathcal{A}$ whenever

$$
\int_{0}^{1}(1-r) \log ^{+} M(r) d r<\infty
$$

Nearly a decade later Hornblower [10] (cf. also [9]) improved MacLane's result, replacing (1.2) with the weaker, and apparently sharp, criterion

$$
\int_{0}^{1} \log ^{+} \log ^{+} M(r) d r<\infty
$$

In addition to establishing the sufficiency of (1.3) he also proved that, corresponding to each $\epsilon>0$, there are functions not in $\mathcal{A}$ for which

$$
\log ^{+} \log ^{+} M(r) \leq \frac{\epsilon}{(1-r) \log \frac{1}{(1-r)}} .
$$

On the other hand, more than fifty years ago Valiron [22] (cf. also [23, p. 191]) had actually shown that Hornblower's examples must have the property that

$$
\limsup _{r \rightarrow 1} \frac{\log \log \log M(r)}{-\log (1-r)} \geq 1
$$

and he further showed that equality in (1.5) is possible, from which it follows that, for any $\epsilon>0$, there are functions not in $\mathcal{A}$ with

$$
\log ^{+} \log ^{+} M(r) \leq \frac{1}{(1-r)^{1+\epsilon}}
$$

Unfortunately, the work of Valiron seems to have been overlooked by subsequent authors and we are grateful to A. E. Eremenko for bringing it to our attention. 
The examples constructed by both Valiron and Hornblower are rather long and quite technically involved. Our primary objective is to present a simple example of the Valiron-Hornblower type based on a kind of $\bar{\partial}$-mollification. Although this work was carried out before we became aware of [22], it is, nevertheless, closer in spirit to the work of Valiron than to that of Hornblower.

Our second objective is to give a new proof of the sufficiency of (1.3) based on an idea of E. M. Dyn'kin [7]. Homblower's original proof in [10] relies (and so indirectly does ours) on a well-known theorem of Beurling, Levinson, Sjöberg and Wolf concerning the existence of a greatest subharmonic minorant to a given function (cf. [1], [2], [12], [13], [21], [25]). A special case of the latter was obtained much carlier by Carleman [4], but his method is quite general in nature. Later MacLane [15] found another approach leading to the sufficiency of (1.3) and in the process gave a new proof of the Beurling-Levinson-Sjöberg-Wolf theorem, which, incidentally; was also discovered by Gurarii [8]. Definitive results in this direction can be found in articles [5] and [6] of Domar (cf. also $[11$, p. 374-383]).

There are, of course, other ways in which to describe or capture the boundary behavior of analytic functions subject to a growth restriction, the most noteworthy being in terms of distribution theory. It follows from the Schwartz program that if $f$ is analytic in $D$ then the functions $f_{r}=f\left(r e^{i \theta}\right)$ converge as $r \uparrow 1$ to a distribution supported on $\partial D$ if and only if there exist constants $C, k$ such that

$$
M(r) \leq \frac{C}{(1-r)^{k}}, \quad r<1
$$

The corresponding result for functions with very rapid growth is due to Beurling [1] and is this: As $r \uparrow 1$ the functions $f_{r}$ converge to a generalized distribution if and only if

$$
\int_{0}^{1} \log ^{+} \log ^{+} M(r) d r<\infty
$$

We do not, at present, know of any direct connection between the existence of asymptotic and distributional boundary values. 


\section{Functions with no asymptotic values}

In this section we shall outline a procedure for constructing analytic functions with a given growth, having no asymptotic boundary values. Later in Section 3 we shall present the more technical details associated with that construction.

To this end let $M(r)$ be a given nondecreasing function defined for $0 \leq r<1$ such that $M(r) \geq e$ and let us assume that

$$
\int_{0}^{1} \log \log M(r) d r=+\infty .
$$

Setting $\mu(r)=\log \log M(1-2 r)$ we obtain a nonincreasing function on $0<r \leq 1 / 2$ with $\int_{0}^{1 / 2} \mu(r) d r=\infty$. With only mild regularity assumptions on $M(r)$ (or equivalently on $\mu(r)$ ) we arrive at our main theorem:

Theorem 1. If $M(r)$ is given as above satisfying (2.1) and if, moreover,

(1) $r \mu(r) \rightarrow 0$ as $r \rightarrow 0$

(2) $\mu(r) \geq c \mu(2 r), 0<r<1 / 2$ and $c>1$

(3) $\int_{0} \frac{1}{r \mu(r)} d r<\infty$

then there exists a function $f$ analytic in $D$, not however belonging to A, such that

$$
M(r, f) \leq M(r), \quad 0 \leq r<1
$$

It should be noted here that $\mu(r)=\frac{1}{r \log 1 / r}$ is a typical function satisfying the conditions of Theorem 1 and it represents the situation studicd by Hornblower [10]. Also, taking $u=\log |f|$ we recover a theorem of Rippon [20] and Hayman [9], since $u$ is subharmonic and, as we shall see, has no asymptotic boundary values on $\partial D$.

As a first step in the proof we define

$$
\chi(y)=\int_{y}^{1 / 2} \mu(t) d t .
$$

Evidently, $\chi$ is a monotonically decreasing function and $\chi(0+)=$ $\lim _{y \downarrow 0} \chi(y)=\infty$. We can, therefore, define $\psi(x)=\chi^{-1}(x)$ for $x \geq 1$ and $\psi$ can be extended to the entire interval $(0,+\infty)$ in such a way that $\psi(0+)=\infty$. The domain $\mathcal{O}$ bounded by the two curves $\psi(x)$ and $\psi(x+2 \pi)$ will be of special interest. 


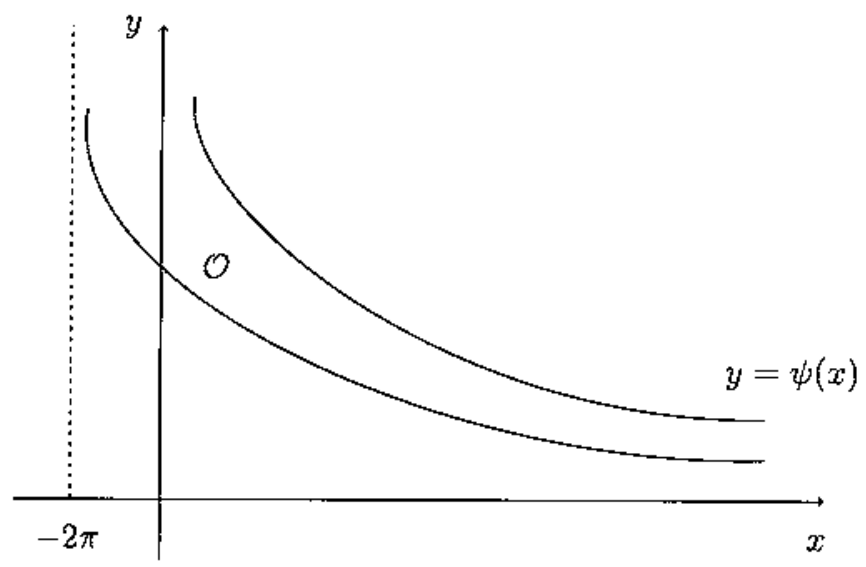

Figure 1

The map $J(w)=e^{i w}$ takes $\mathcal{O}$ onto $\Omega=D \backslash \Gamma$, where

$$
\Gamma:|z|=e^{-\psi(\arg z)}
$$

is a spiral asymptotic to $\partial D$. If we now choose a conformal map $G: \mathcal{O} \rightarrow$ $\Pi=\{\zeta=\xi+i \eta:|\eta|<\pi / 2\}$ with $G(+\infty)=+\infty$ we can, in principle, look for functions with a given growth in $\Pi$ and by transferring them to $\Omega$ we might hope to obtain the desired example.

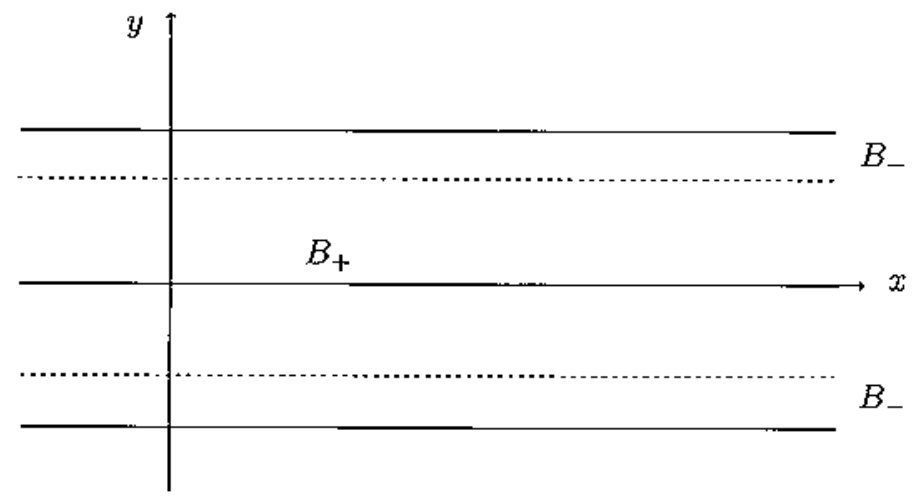

Figure 2 
Fix $a>1$, define $l_{a}(\zeta)=\exp \exp (a \zeta)$ for $\zeta \in \Pi$, and consider the following two regions:

(i) $B_{+}=\left\{\zeta \in \Pi:|\eta|<\frac{\pi}{2 a}\right\}$

(ii) $B_{-}=\left\{\zeta \in \Pi: \frac{\pi}{2 a}+\epsilon<|\eta|<\frac{\pi}{2}\right\}, \epsilon$ small and positive.

The function

$$
L_{a}(z)=l_{a}(G(-i \log z)), \quad z \in \Omega
$$

is defined on $\Omega$ and grows like $l_{a}$ in the corresponding spiral domains $S_{ \pm}=J \circ G^{-1}\left(B_{ \pm}\right)$. In particular, if $a=1+\epsilon$ is close to 1 then

(iii) $L_{a}(z) \rightarrow \infty$ as $z \rightarrow \partial D, z \in S_{+}$

(iv) $L_{a}(z) \rightarrow 0$ as $z \rightarrow \partial D, z \in S_{-}$.

Hence, $L_{a}$ can have no asymptotic values at $\partial D$. However, it has jump discontinuities all along $\Gamma$ and so does not directly suite our purposc. Our main task will be to construct an analytic function sufficiently close to $L_{a}$ that it inherits the same behavior in $S_{+}$and $S_{-}$.

Step 1. We begin by mollifying $L_{a}$ in the following way: For each $z \in D$ let $D_{z}$ be the disk with center at $z$ and radius $\beta(1-|z|)$. Here, $\beta$ is a small, but fixed, positive constant to be specified later. Next consider the average

$$
F(z)=\frac{1}{\left[D_{z}\right]} \int_{D_{z}} L_{a}(\zeta) d A_{\zeta}
$$

Although $F$ is still not analytic in $D$, if $\beta$ is chosen properly we can arrange that

(4) $F(z)=L_{0}(z)$ for $z \in \Gamma_{0}=J \circ G^{-1}(\mathbb{R})$

(5) $|F(z)| \leq C M(|z|)$

(6) $|\bar{\partial} F(z)| \leq$ const $<\infty$.

Because $F(z) \rightarrow \infty$ as $z \rightarrow \partial D$ along $\Gamma_{0}$ and $|F(z)|$ is bounded on $\Gamma$, there can again be no asymptotic values on $\partial D$.

Step 2 consists in selecting an analytic component of $F$ defined by setting

$$
A(z)=F(z)-\frac{1}{\pi} \int_{D} \frac{\bar{\partial} F(\zeta)}{\zeta-z} d A_{\zeta}
$$

Since, by virtue of property (6), $|F-A| \leq$ const $<\infty$ all the requisite growth restrictions are preserved and $A(z)$ is the function we are looking for. 


\section{Technicalities of the construction}

Our task here is to verify assertions (4), (5) and (6) of Section 2. And, we can assume without loss of generality that

(i) $-y \mu^{\prime}(y) \geq c \mu(y), c>0$

for some positive $c$. Otherwise, we could simply replace $\mu(y)$ by

$$
\mu_{1}(y)=\int_{y}^{2 y} \frac{\mu(t)}{t} d t
$$

taking care to check that $\mu(y)$ and $\mu_{1}(y)$ are comparable and that (i) is satisfied by virtue of property (2). Let us also recall that $\psi=\chi^{-1}$, so that if $y=\psi(x)$, then by (I)

$$
\frac{\psi^{\prime}(x)}{\psi(x)}=\frac{-1}{y \mu(y)} \rightarrow-\infty
$$

as $x \rightarrow \infty$ or, equivalently, as $y \rightarrow 0$. Hence, $\frac{\psi(x+2 \pi)}{\psi(x)} \rightarrow 0$ as $x \rightarrow \infty$ and it follows that for any $\epsilon>0$,

$$
\theta(x) \leq \psi(x) \leq(1+\epsilon) \theta(x), \quad x \geq x(\epsilon)
$$

where $\theta(x)=\psi(x)-\psi(x+2 \pi)$ is the vertical width of $\mathcal{O}$ at the point $x$.

To establish property (4) it is sufficient to prove that if $z \in \Gamma_{0}$ then the disk $D_{z}$ of radius $\beta(1-|z|)$ does not meet $\Gamma$ if $\beta$ is small (and fixed) and $z$ is near $\partial D$. To this end let us suppose that $z \in \Gamma_{0}$ and let $\zeta=G(-i \log z)$ be the corresponding point in $\Pi$, which, of course, lies on the real axis $\mathbb{R}$. Denote by $Q$ the family of curves lying in $\Pi$ and separating $\zeta$ from $\eta=\pi / 2$, that is, from the top; $Q^{*}$ is the conjugate family defined relative to the bottom. The length-width ratio of $Q$ is

$$
\rho(Q)=\frac{l(Q)}{l\left(Q^{*}\right)}
$$

where $l(Q)$ and $l\left(Q^{*}\right)$ denote the infimum of the lengths of the rectifiable members of $Q$ and $Q^{*}$, respectively. Evidentiy, $\rho(Q)$ is bounded and bounded away from zero as $\zeta \rightarrow+\infty$ along $\mathbb{R}$. Since this is a conformally invariant property of $\rho$, (cf. McMillan [17], [18]), it follows from (3.1) that $\beta$ can be chosen as indicated; hence (4). 
To confirm assertion (5) we appeal to a theorem of Warshawski (cf. $[24$, p. 296] and [9, p. 761]) according to which

$$
\operatorname{Re} G(-i \log z)=\pi \int_{1}^{x(\log 1 /|z|)} \frac{d t}{\theta(t)}+C+O(1)
$$

as $z \rightarrow \partial D$, since property (3) assures that the crucial assumption

$$
\begin{aligned}
\int^{\infty} \frac{\psi^{\prime}(x)^{2}}{\theta(x)} d x+\int^{\infty} \frac{\psi^{\prime}(x+2 \pi)^{2}}{\theta(x)} d x \leq k \int^{\infty} \frac{\psi^{\prime}(x)^{2}}{\psi(x)} d x= \\
=k \int_{0} \frac{d y}{y \mu(y)}<\infty
\end{aligned}
$$

is satisfied. Note that (3.1) has also been used. Hence, it follows that for $z$ near $\partial D$

$$
\left|L_{a}(z)\right|=|\exp \exp a G(-i \log z)| \leq \exp \exp \left(\pi a \int_{1}^{\chi^{(\log 1 /|z|)}} \frac{d t}{\theta(t)}+C a\right)
$$

and, again taking (i) and (3.1) into account, we conclude that

$$
\begin{aligned}
\left|L_{\Omega}(z)\right| & \leq \exp \exp \left(K \int_{1}^{x(1 / 2(1-|z|)} \frac{d t}{\psi(t)}\right) \\
& \leq \exp \exp K \mu(1 / 2(1-|z|)) \\
& \leq M(|z|) .
\end{aligned}
$$

The constants $K, C$ are, of course, absolute and with no loss of generality we have assumed that $K=1$. Consequently, these estimates and the fact that

$$
|F(z)| \leq \sup _{|\zeta-z| \leq \beta(1-|z|)}\left|L_{a}(\zeta)\right|
$$

lead directly to the desired inequality (5).

Thus, it remains only to prove (6) or, equivalently, that $|\bar{\partial} F(z)| \leq$ $K<\infty$ throughout $D$. To accomplish this we first express $L_{a}$ as the sum of an analytic function $H$ and the Cauchy integral of a measure $\nu$ supported on $\Gamma$ :

$$
L_{a}(\zeta)=H(\zeta)+\frac{1}{2 \pi i} \int_{\Gamma} \frac{d \nu(t)}{t-\zeta} .
$$

The important thing to note here is that $d \nu(z)=j(z) d z$ where, for each $z \in \Gamma$, the density

$$
j(z)= \pm\left(L_{a}\left(z^{+}\right)-L_{a}\left(z^{-}\right)\right)
$$


is the jump of $L_{a}$ across $\Gamma$, the sign being chosen to agree with the orientation of $\Gamma$. Introducing the kernel

$$
K(z, t)=\frac{1}{\left|D_{z}\right|} \int_{D_{z}} \frac{d A_{\zeta}}{t-\zeta}
$$

and integrating (3.6) with respect to area it follows that

$$
F(z)=H(z)+\frac{1}{2 \pi i} \int_{\Gamma} K(z, t) d \nu(t) .
$$

On the other hand, it is a relatively simple matter to check that

$$
K(z, t)= \begin{cases}\frac{1}{t-z}, & t \notin D_{z} \\ \frac{\pi(t-z)}{\left|D_{z}\right|}, & t \in D_{z}\end{cases}
$$

from wbich we easily conclude that

$$
|\bar{\partial} F(z)| \leq \frac{1}{2 \pi} \int_{\Gamma}|\bar{\partial} K(z, t)| d|\nu|(t) \leq \frac{|\nu|\left(D_{z}\right)}{\left|D_{z}\right|} .
$$

The last expression vanishes unless $D_{z} \cap \Gamma \neq \phi$ and so we have the estimate

$$
|\bar{\partial} F(z)| \leq \begin{cases}\frac{1}{\left|D_{z}\right|} \sup _{\zeta \in D_{z} \cap \Gamma}\left|L_{a}(\zeta)\right|: & \text { if } D_{z} \cap \Gamma \neq \phi \\ 0, & \text { otherwise. }\end{cases}
$$

And, moreover, when $\zeta \in \Gamma$ Warshawski's estimate (3.2) takes the particularly convenient form

$$
\begin{aligned}
\left|L_{a}(\zeta)\right|=\mid & \exp \exp a G(-i \log \zeta) \mid \leq \\
& \leq \exp \left\{-\left|\cos \frac{\pi}{2} a\right| \cdot \exp \left(\pi a \int_{1}^{x(\log 1 /|\zeta|)} \frac{d t}{\theta(t)}+C a\right)\right\}
\end{aligned}
$$

From this it follows easily that if $\zeta \in \Gamma$ then

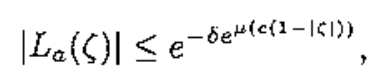

where $\delta=\left|\cos \frac{\pi}{2} a\right|$ and $c>0$ is suitably chosen, since by (3.1)

$$
\begin{aligned}
\int_{1}^{\chi(\log 1 /|\zeta|)} \frac{d t}{\theta(t)} \geq \int_{1}^{\chi(2(1-|\zeta|)} & \frac{d t}{\psi(t)}> \\
& >\int_{1-|\zeta|}^{2(1-|\zeta|)} \frac{\mu(s)}{s} d s \geq \mu(2(1-|\zeta|)) \cdot \log 2
\end{aligned}
$$


provided, of course, that $\zeta$ is close to $\partial D$. Therefore, adjusting the constant in (3.8) to a account for the fact that $z \in D_{z}$, but perhaps $z \notin \Gamma$, we conclude that

$$
|\ddot{\partial} F(z)| \leq \frac{1}{(1-|z|)} e^{-\delta e^{\mu(c(1-|z|))}}
$$

everywhere in $D$, and so is trivially bounded, since $\mu(r) \sim 1 / r^{\mathrm{c}}$ as $r \rightarrow 0$ by way of assumption (i).

\section{Functions belonging to class $\mathcal{A}$}

Our goal here is to give a short proof of the following theorem of Hornblower [10].

Theorem 2. Let $f(z)$ be analytic and nonconstant in $D$ and let $M(r)=\sup _{|z|=r}|f(z)| . I f$

$$
\int_{0}^{1} \log ^{+} \log ^{+} M(r) d r<\infty
$$

then $f \in \mathcal{A}$.

All known proofs, including Hornblower's, are based on a result of Levinson [12, p. 135], concerning the growth of harmonic measure in a cusp (cf. also Beurling [2, p. 381]). We are able to avoid any mention of harmonic measure by making use of the following result of Dyn'kin, the details of which can be found in [7].

Lemma. Let $M(r)$ be a monotonically increasing function defined for $0 \leq r<1$ such that (4.1) is satisfied and let $\gamma^{\prime}, \gamma$ be two subarcs of $\partial D$ with $\gamma^{\prime} \subseteq \gamma$. Then there exists a function $\phi \in C^{1}$ such that

(1) $\phi \equiv 1$ on $\gamma^{\prime}$

(2) $\phi \equiv 0$ outside a neighborhood of $\gamma$

(3) $|\bar{\partial} \phi(z)| \leq \frac{1}{M(|z|)}, z \in D$.

To prove Theorem 2 let us suppose that $f$ is analytic in $D$ and that (4.1) is satisfied. Assuming that $f \notin \mathcal{A}$ there is an arc $\gamma \subseteq \partial D$, no point of which is the endpoint of an asymptotic path for $f$. It follows, without loss of generality, from our remarks in Section 1 (cf. (ii)) that there exists a sequence of Koebe arcs $\gamma_{n} \rightarrow \gamma$ such that $f$ is bounded on 
U $\gamma_{n}$. Now choose $\gamma^{\prime} \subseteq \gamma$ and corresponding function $\phi$ as in Dyn'kin's lemma. Extend each

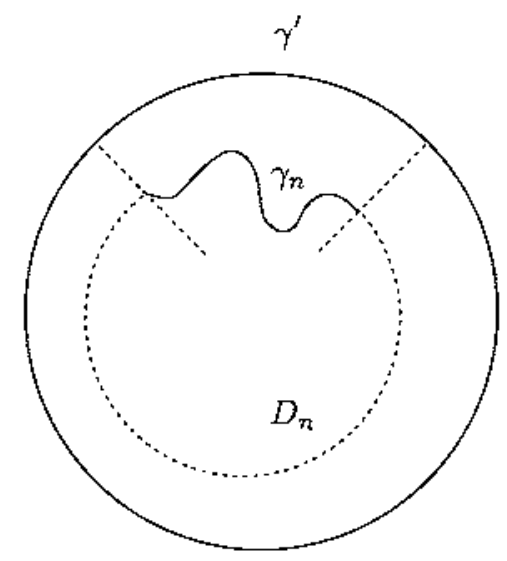

Figure 3

arc $\gamma_{n}$ to a simple closed curve enclosing a region $D_{n}$ in such a way that $D_{n} \rightarrow D$ as $n \rightarrow \infty$. For each $n=1,2, \ldots$ let $g_{n}: D_{n} \rightarrow D$ be a conformal map with $g_{n}(0)=0$. Applying Green's theorem to $f g_{n}^{\prime} \phi$ we conclude that, for each $z$ in some fixed neighborhood of $\gamma^{\prime}$,

$$
\begin{aligned}
\left|f(z) g_{n}^{\prime}(z) \phi(z)\right| \leq \frac{1}{\pi} \int_{D_{n}} & \frac{|\bar{\partial} \phi(\zeta)|\left|f g_{n}^{\prime}(\zeta)\right|}{|\zeta-z|} d A_{\zeta}+ \\
& +\limsup _{n \rightarrow \infty} \int_{\gamma_{n}} \frac{|\phi(\zeta)||f(\zeta)|}{|\zeta-z|}\left|g_{n}^{\prime}(\zeta) \| d \zeta\right|+\text { const. }
\end{aligned}
$$

from which it follows that $\left|f(z) g_{n}^{\prime}(z)\right| \leq C_{1}(1-|z|)^{-1}+C_{2}$ in a neighborhood of $\gamma^{\prime}$, since $\int_{\gamma_{n}}\left|g_{n}^{\prime}(\zeta) \| d \zeta\right| \leq 2 \pi$ and, by the Koebe distortion theorem, $\int_{D_{n}}\left|g_{n}^{\prime}(\zeta)\right|^{2+\varepsilon} d A \leq M$ with $M$ depending only on diam $\left(D_{n}\right)$. On the other hand, the Carathéodory convergence theorem implies that $\left|g_{n}^{\prime}\right| \rightarrow 1$ pointwise and boundedly almost everywhere in $D$, and therefore

$$
|f(z)| \leq C_{1}(1-|z|)^{-1}+C_{2}
$$

in a neighborhood of $\gamma^{\prime}$. This, together with the boundedness of $f$ on $\cup \gamma_{n}$, implies immediately that $f$ is in fact bounded near $\gamma^{\prime}$. To see this just choose $\lambda_{1}, \lambda_{2} \in \gamma^{\prime}$ and note that $\left(z-\lambda_{1}\right)\left(z-\lambda_{2}\right) f$ is bounded. As a result $f$ must have radial limits almost everywhere on $\gamma^{\prime}$, contrary to assumption. Therefore, $f \in \mathcal{A}$. 


\section{References}

1. A. Betrling, "Quasianalyticity and general distributions," Lecture notes, Standforf Univ., 1961.

2. A. Beurling, "Collected works," vol. 1, Birkhäuser, Boston, 1989.

3. T. Carleman, Sur les fonctions inverses des fonctions entières d'ordre fini, Ark. Mat. Astron. och Fysik 15 (1920), 1-7.

4. T. Carlfman, Extension d'un théoréme de Liouville, Acta Moth. 48 (1926), 363-366.

5. Y. DOMAR, On the existence of a largest subharmonic minorant of a given function, Ark. Mat. 3 (1957), 429-440.

6. Y. DOMaR, Uniform boundedness in families related to subharmonic functions, J. London Math. Soc. 38 (1988), 485491.

7. E. M. DYN'KIN, Functions with a given estimate for $\frac{\partial f}{\partial \bar{z}}$ and N. Levinson's Theorem, Mat. Sb. 89 (1972), 182-190; Math. USSR-Sb. 18 (1972), 181-189:

8. V. P. GURARII, On a theorem of N. Levinson concerning normal families of analytic functions, Zap. Nauchn. Sem. Leningrad Otdel Mat. Inst. Steklov (LOMI) 19 (1970), 215-220.

9. W. K. Hayman, "Subharmonic functions," vol. 2, Academic Press, London, 1989.

10. R. HoRnblower, A growth condition for the Maclane class $\mathcal{A}$, Proc. London Math. Soc. 23 (1971), 371-384.

11. P. Koosis, "The logarithmic integral," Cambridge Univ. Press, Cambridge, 1988.

12. N. Levinson, An inequality in function theory, Bull. Amer. Math. Soc. 45 (1939), p. 236.

13. N. Levinson, Gap and density theorems, Amer. Math. Soc. Collog. Publ. 26 (1940), New York.

14. G. R. MacLane, Asymptotic values of holomorphic functions, Rice Univ. Studies 49, no.1 (1963).

15. G. R. Maclane, A growth condition for class $\mathcal{A}$, Michigan Math. J. 25 (1978), 263-287.

16. J. E. MCMillan, Asymptotic values of functions holomorphic in the unit disk, Michigan Math. J. 12 (1965), 141-154.

17. J. E. MCMILLAN, Distortion under conformal and quasiconformal mappings, Acta Math. 126 (1971), 121-141.

18. J. E. MCMillan, "Boundary behavior under conformal mapping," Proc. NRL Conference on Classical Function Theory, Washington, 
D.C., 1970.

19. R. NevanlinNa, "Analytic Functions," Springer-Verlag, Berlin, 1970 .

20. P. I. Rippon, On a growth condition related to the MacLane class, J. London Math. Soc. 18 (1978), 94-100.

21. N. SjöBerg, Sur les minorantes sousharmoniques d'une fonction donée, Neuvième Congres des Math. Scand., Helsinki (1938), 309-319.

22. G. VAliron, Sur les singularitiés de certaines fonctions holomorphes et de leurs inverses, J. Math. Pures Appl. 15 (1936), 423-435.

23. G. Valiron, "Fonctions analytiques," Presses Universitairés de France, 1954.

24. S. E. WARSHAWSKI, On conformal mapping of infinite strips, Thans. Amer. Math. Soc. 51 (1942), 280-335.

25. F. WOLF, On majorants of subharmonic and analytic functions, Bull. Amer. Math. Soc. 45 (1942), 925-932.

J. E. Brennar:

Department of Mathematics

University of Kentucky

Lexington, Ky. 40506

U.S.A.
A. L. Volberg:
Department of Mathematics
Michigan State University
East Lansing, MI 48824
C.S.A.

Rebut el 6 de Juliol de 1993 